\title{
Instantaneous Bethe-Salpeter Approach to Pseudoscalar Mesons
}

\section{Wolfgang Lucha*}

Institute for High Energy Physics, Austrian Academy of Sciences, Nikolsdorfergasse 18, A-1050

Vienna, Austria

E-mail: Wolfgang. Lucha@oeaw.ac.at

Light pseudoscalar mesons are Janus-type particles: Within quantum chromodynamics, they must be described as bound states of its fundamental degrees of freedom and as the (pseudo-) Goldstone bosons of its spontaneously broken chiral symmetry. This janiform nature of pions and kaons may be easily accommodated by the Bethe-Salpeter formalism in its instantaneous limit: Starting from the general shape of the Bethe-Salpeter solutions for light pseudoscalar mesons at large Euclidean momenta, we provide the exact relationship between the solutions of our bound-state equation and the underlying interactions, boiled down to potentials $V(r)$ depending on the interquark distance $r$. For massless quarks, $V(r)$ exhibits, at the origin, a (logarithmically softened) Coulomb singularity crucial for counterbalancing all positive contributions to the bound-state mass but rises, for large $r$, to infinity and can hence be regarded as confining. For massive quarks, $V(r)$ still features a similar (logarithmically softened) Coulomb singularity at the origin; for quark masses too large, however, the potential's confining character gets lost: $V(r)$ approaches, for large $r$, a nonpositive finite limit.
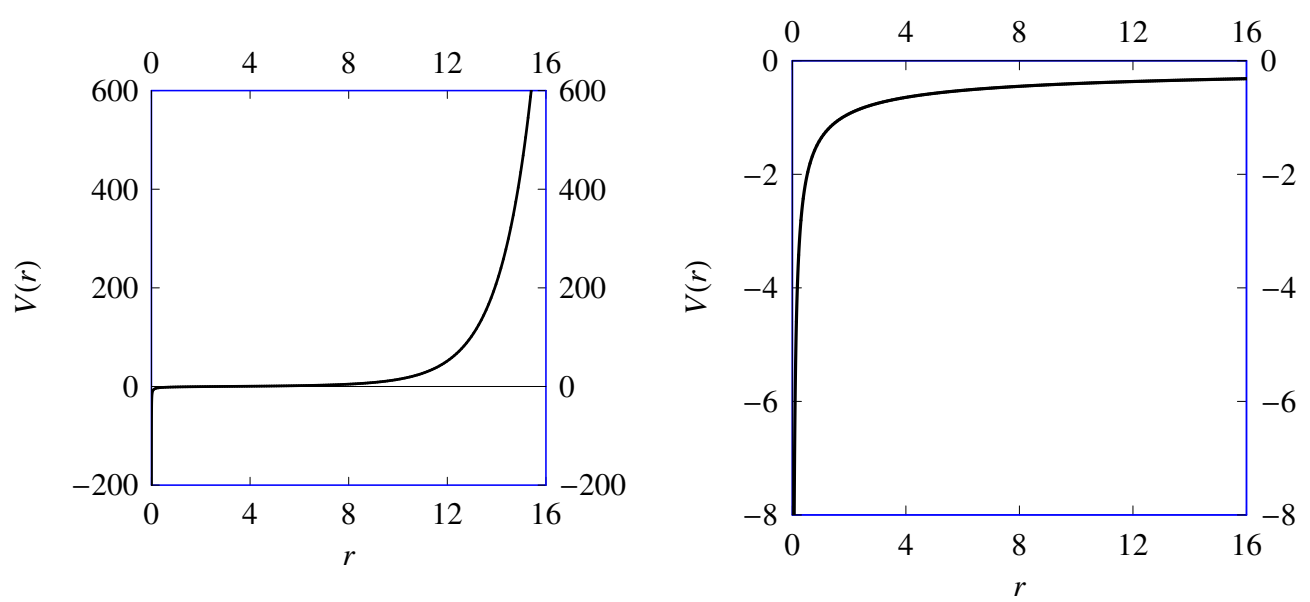

The European Physical Society Conference on High Energy Physics - EPS-HEP2013

18-24 July 2013

Stockholm, Sweden

\footnotetext{
* Speaker.
} 


\section{Introduction, Cursory Sketch of Basic Idea, Summary of Findings, and Conclusions}

In principle, the homogeneous Bethe-Salpeter equation [1] provides a quantum-field-theoretic description of relativistic bound states in Minkowski space. In real life, practical or even conceptual obstacles often prompt us to content ourselves with three-dimensional reductions of this formalism. Most prominent among the resulting bound-state equations is the Salpeter equation [2], obtained by assuming that all bound-state constituents interact instantaneously and propagate freely, cf. Ref. [3]. An astounding drawback of the Salpeter equation is that, depending on the nature of the interactions involved, it predicts, even in situations where we expect to obtain just stable bound states, also states that develop instabilities. This issue has been thoroughly analysed for the Salpeter equation without negative-energy contributions and a generalization [4] thereof [5], and the full Salpeter equation [6]. As a by-product, such studies bore out the need for exact analytic solutions of the Salpeter equation.

Examples of these may be found by inverting the procedure: Assuming spherical symmetry, all interactions are encoded in configuration-space central potentials and Salpeter's equation reduces to systems of radial eigenvalue equations [7]. Then rigorous relations between the properties of bound states and the interactions which their constituents experience can by established by determining for pre-selected solutions those potentials for which the bound-state equation yields these solutions [8].

Naturally, the question arises how that relation looks like for light pseudoscalar mesons viewed as - due to (explicitly and) spontaneously broken global symmetries of quantum chromodynamics (almost) massless - quark-antiquark bound states. In the chiral limit of quantum chromodynamics with dynamically broken chiral symmetry, pseudoscalar-meson Bethe-Salpeter solutions [9] fall off (for large Euclidean momenta) like the inverse fourth power of the quarks' relative four-momentum. Choosing our Salpeter model according to these insights and Fierz-symmetric effective interactions of the quarks then gives the central potentials for massless and massive quarks in analytic form [10].

\section{References}

[1] H. A. Bethe and E. E. Salpeter, Phys. Rev. 82 (1951) 309; M. Gell-Mann and F. Low, Phys. Rev. 84 (1951) 350; E. E. Salpeter and H. A. Bethe, Phys. Rev. 84 (1951) 1232.

[2] E. E. Salpeter, Phys. Rev. 87 (1952) 328.

[3] W. Lucha, F. F. Schöberl, and D. Gromes, Phys. Rep. 200 (1991) 127; W. Lucha and F. F. Schöberl, Int. J. Mod. Phys. A 14 (1999) 2309; Fizika B 8 (1999) 193.

[4] W. Lucha and F. F. Schöberl, J. Phys. G 31 (2005) 1133; AIP Conf. Proc. 892 (2007) 524; Z.-F. Li, W. Lucha, and F. F. Schöberl, Mod. Phys. Lett. A 21 (2006) 1657.

[5] W. Lucha and F. F. Schöberl, AIP Conf. Proc. 964 (2007) 318; Frascati Phys. Ser. 46 (2007) 1539; Z.-F. Li, W. Lucha, and F. F. Schöberl, Phys. Rev. D 76 (2007) 125028; J. Phys. G 35 (2008) 115002.

[6] W. Lucha, PoS(Confinement8)164 (2009); AIP Conf. Proc. 1317 (2010) 122; 1343 (2011) 625.

[7] J.-F. Lagaë, Phys. Rev. D 45 (1992) 305; M. G. Olsson, S. Veseli, and K. Williams, Phys. Rev. D 52 (1995) $5141 ; 53$ (1996) 504.

[8] W. Lucha and F. F. Schöberl, Phys. Rev. D 87 (2013) 016009.

[9] P. Maris, C. D. Roberts, and P. C. Tandy, Phys. Lett. B 420 (1998) 267; P. Maris and C. D. Roberts, Phys. Rev. C 56 (1997) 3369.

[10] W. Lucha and F. F. Schöberl, in preparation. 